Europhysics Letters

PREPRINT

\title{
Shape oscillation of a rotating Bose-Einstein condensate
}

\author{
Sabine Stock, Vincent Bretin, Frédéric Chevy and Jean Dalibard \\ Laboratoire Kastler Brossel(*), 24 rue Lhomond, F-75231 Paris Cedex 05, France
}

PACS. 03.75.Fi - Phase coherent atomic ensembles; quantum condensation phenomena. PACS. 32.80.Pj - Optical cooling of atoms; trapping.

\begin{abstract}
We present a theoretical and experimental analysis of the transverse monopole mode of a fast rotating Bose-Einstein condensate. The condensate's rotation frequency is similar to the trapping frequency and the effective confinement is only ensured by a weak quartic potential. We show that the non-harmonic character of the potential has a clear influence on the mode frequency, thus making the monopole mode a precise tool for the investigation of the fast rotation regime.
\end{abstract}

The investigation of rotating gases or liquids is a central issue in the study of superfluids 12 . During the recent years, several experiments using rotating atomic Bose-Einstein condensates have provided a spectacular illustration of the notion of quantized vortices $3,4,5,6$. Depending on the rotation frequency of the gas, a single vortex or a regular array of vortices can be observed experimentally. When the rotation frequency is increased to a very large value, a new class of phenomena is predicted, in connection with quantum Hall physics 7, $8,9,10,11,12,13,14$. For a gas confined in a harmonic potential, the fast rotation domain corresponds to stirring frequencies $\Omega$ of the order of the trapping frequency $\omega_{\perp}$ in the plane perpendicular to the rotation axis (hereafter denoted $z$ ). From a classical point of view, the transverse trapping and centrifugal forces compensate each other for this stirring frequency, and the motion of the particles in the $x y$ plane is only driven by Coriolis and interatomic forces. This situation is similar to that of an electron gas in a magnetic field, since Lorentz and Coriolis forces have the same mathematical structure.

In order to approach the regime of fast rotation two paths are currently being explored. The first approach is implemented in a pure harmonic potential and is based on evaporative spin-up, i.e. the selective removal of particles with low angular momentum [15. The stirring frequency $\Omega$ can then be raised close to $\omega_{\perp}\left(\Omega=0.993 \omega_{\perp}\right.$ was reached in [16]). The second approach, which is followed here, consists in adding to the quadratic confinement a small positive quartic potential, which ensures that the particles will remain confined even when $\Omega$ exceeds $\omega_{\perp} 1718,19202122$. We have recently proven that this method can be successfully implemented and we have mechanically stirred a rubidium Bose-Einstein condensate up to $\Omega \simeq 1.05 \omega_{\perp}[23$.

All experiments performed so far (including the present one) are still deeply within the mean field regime, characterized by a number of vortices $N_{v}$ well below the number of particles

$\left(^{*}\right)$ LKB is a unité de recherche of Ecole Normale Supérieure and Université Paris 6, associated to the CNRS.

(c) EDP Sciences 
$N$. Nevertheless in order to prepare for future investigations of possible quantum-Hall-like states, one must design proper tools of investigation, such as the study of the eigenmodes of the rotating gas. Possible examples are the transverse quadrupole modes, which allow for a measurement of the $z$ component of the angular momentum [23], and the Tkachenko oscillations of the vortex lattice, recently observed in [15]. Here we report on the experimental and theoretical study of the lowest transverse monopole mode of an ultra-cold gas of rubidium atoms in the fast rotation regime.

In a trap with axial symmetry along the $z$-axis, excitations can be characterized by their angular momentum along $z$ (quantum number $m_{z}$ ) [24. Here we are interested in excitations carrying no angular momentum $\left(m_{z}=0\right)$. In the limit $\omega_{z} \ll \omega_{\perp}$ which is of interest here, it is possible to identify $m_{z}=0$ "transverse monopole" modes, which mostly affect the atom distribution in the $x y$ plane. For a non-rotating condensate the lowest frequency for these $m=0$ transverse modes is $\omega_{\mathrm{mp}} \simeq 2 \omega_{\perp}$ (transverse breathing mode) 25 26]. In the present work we show experimentally that the relation $\omega_{\mathrm{mp}} \simeq 2 \omega_{\perp}$ remains valid for large rotation frequencies (as predicted by [27]), as long as the quartic term in the confinement is not significant. We also explore the region $\Omega \sim \omega_{\perp}$, where the quartic term plays an essential role. We compare the measured monopole frequency with that derived from a simple hydrodynamic model of an infinite, cylindrical condensate. Finally we discuss the structure of the mode, which exhibits a very particular behavior in the range $\Omega \gtrsim \omega_{\perp}$.

Our ${ }^{87} \mathrm{Rb}$ Bose-Einstein condensate contains $3 \times 10^{5}$ atoms. It is produced by radiofrequency evaporation in a Ioffe-Pritchard magnetic trap, to which we superimpose a far-blue detuned laser beam. The magnetic trap provides a harmonic confinement with cylindrical symmetry along the $z$ axis, with $\omega_{z} / 2 \pi=11.0 \mathrm{~Hz}$ and $\omega_{\perp}^{(0)} / 2 \pi=75.5 \mathrm{~Hz}$. The laser beam adds a negative quadratic and a positive quartic potential in the $x y$ plane. The quadratic term decreases the trap frequency $\omega_{\perp}$ by $\sim 15 \%$. The quartic term allows to explore rotation frequencies around and slightly above the trapping frequency $\omega_{\perp}$. It reads $k r^{4} / 4$, with $r^{2}=$ $x^{2}+y^{2}$ and $k=2.6(3) \times 10^{-11} \mathrm{~J} / \mathrm{m}^{4}$. The oscillation frequency of the condensate centerof-mass (dipole motion) in the $x y$ plane is $\omega_{\mathrm{dp}} / 2 \pi=65.6( \pm 0.3) \mathrm{Hz}$. Because of the quartic component of the trapping potential, $\omega_{\mathrm{dp}}$ is slightly larger than $\omega_{\perp}$, even for an arbitrarily small amplitude of the dipole motion. The condensate has indeed a finite radius $R_{c} \simeq 6.5 \mu \mathrm{m}$ and therefore explores regions of space where the contribution of the quartic term is significant. Using a perturbative treatment of the quartic term we infer that $\omega_{\mathrm{dp}}-\omega_{\perp} \simeq 2 k R^{2} /\left(7 m \omega_{\perp}^{2}\right)$, so that $\omega_{\perp} /(2 \pi)=64.8( \pm 0.3) \mathrm{Hz}$.

The procedure for setting the gas in rotation at a frequency $\Omega \sim \omega_{\perp}$ has been described in detail in [23]. We apply an additional laser beam creating a rotating anisotropy in the $x y$ plane for two successive phases, with respective durations of $300 \mathrm{~ms}$ and $600 \mathrm{~ms}$. During the first phase the rotation at frequency $\Omega \sim \omega_{\perp} / \sqrt{2}$ resonantly excites the transverse quadrupole mode, and vortices subsequently penetrate the condensate. After a 400 ms relaxation period we apply the second stirring phase at the final desired frequency. This phase is followed by a $500 \mathrm{~ms}$ relaxation period. By measuring the transverse size of the condensate, we have checked that it is then rotating at a frequency close to the stirrer frequency $\Omega$ (within $2 \%$ ) for the regime of interest $\Omega \leq 1.05 \omega_{\perp}$. In particular, for $\Omega \lesssim \omega_{\perp}$ we observe large vortex arrays involving up to $\sim 50$ vortices. During the stirring and relaxation periods, we apply radio frequency (r.f.) evaporative cooling. The r.f. is set $24 \mathrm{kHz}$ above the value which removes all particles from the trap. Assuming $\Omega=\omega_{\perp}$, so that the transverse confinement is purely quartic, this gives a trap depth $U_{0} \sim 40 \mathrm{nK}$, hence a temperature $T \lesssim 10 \mathrm{nK}$.

We then excite the transverse monopole mode(s) of the gas by changing for a period $\tau_{0}$ the intensity of the laser creating the quartic potential from $I$ to $I^{\prime}$. We choose $\tau_{0}=2 \mathrm{~ms}$, which 


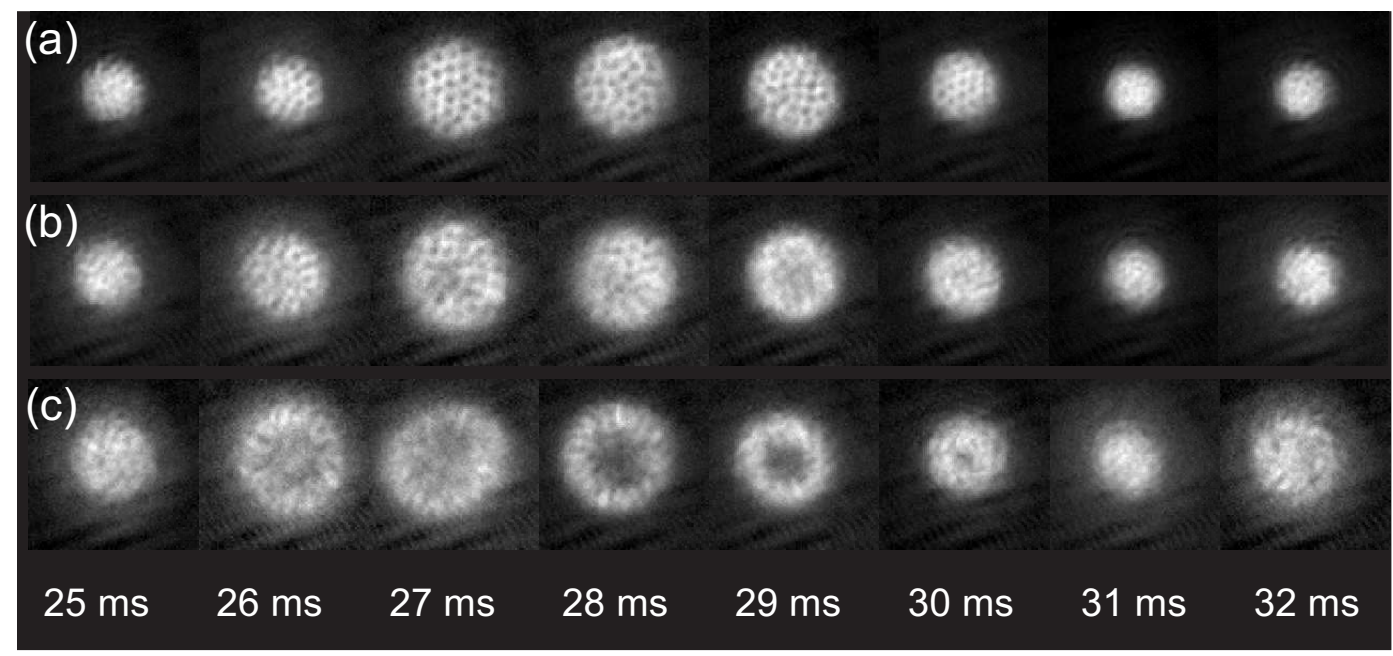

Fig. 1 - Series of images of the condensate for various waiting times $\tau$ after excitation. Row a) corresponds to a rotation frequency $\Omega /(2 \pi)=62 \mathrm{~Hz}$, row b) to $\Omega /(2 \pi)=64 \mathrm{~Hz}$ and row c) to $\Omega /(2 \pi)=66 \mathrm{~Hz}$.

is short compared to the oscillation period of the monopole oscillation $2 \pi / \omega_{\mathrm{mp}}=7.5 \mathrm{~ms}$. We then wait for an adjustable duration $\tau$ before performing a time-of-flight expansion and absorption imaging. The images are taken along the rotation axis $z$ so that we have access to the column density in the $x y$ plane.

Typical results are shown in figure 1 for $\Omega / 2 \pi=62,64,66 \mathrm{~Hz}$. Each image corresponds to a destructive measurement of the atom density after a duration $\tau=25, \ldots, 32 \mathrm{~ms}$ and a $18 \mathrm{~ms}$ time-of-flight. In order to check that the oscillation is in the linear regime, we explored values between 0 and 4 for the ratio $I^{\prime} / I$ : we found that the frequency $\omega_{0}$ is (within experimental uncertainties) independent of the excitation strength $\left|I-I^{\prime}\right| / I$, and that the amplitude of the oscillation varies linearly with this strength. We have also varied the duration of the time-offlight between 6 and $18 \mathrm{~ms}$. We thus verify that all features reported here are independent of this duration, which corresponds to a mere scaling of the initial spatial density distribution.

We have taken similar sequences of images for various stirring frequencies $\Omega$, with $\tau$ varying from 0 to $40 \mathrm{~ms}$ by steps of $1 \mathrm{~ms}$. We extract from these images the transverse size $R(\tau)$ of the gas. The variations of $R(\tau)$ are well fitted by a single sinusoidal function, with frequency $\omega_{\mathrm{mp}}$. The characteristic lifetime of the excitation is larger than $100 \mathrm{~ms}$, so that its decay is negligible during the $40 \mathrm{~ms}$ period. The variations of $\omega_{\mathrm{mp}}$ as a function of $\Omega$ are plotted in figure 2. An obvious feature of this plot is that $\omega_{\mathrm{mp}}$ varies very weakly for $\Omega$ except in the vicinity of $\omega_{\perp}$. We now discuss in more details three relevant domains of this graph.

(1) For a non rotating gas $(\Omega=0)$ we find $\omega_{\mathrm{mp}} /(2 \pi)=130.6( \pm 1.5) \mathrm{Hz}$, which is close to the well known result $\omega_{\mathrm{mp}} \simeq 2 \omega_{\perp}=2 \pi \times 129.6( \pm 0.6) \mathrm{Hz}$ [28].

(2) When the gas rotates at a frequency $\Omega$ not too close to $\omega_{\perp}$, the effect of the quartic potential remains small. In these conditions we find that the monopole frequency $\omega_{0}$ stays approximately constant. At first sight this may seem a surprising result. For a rotating gas in a pure harmonic potential, the transverse confinement is reduced due to the centrifugal force. In the calculation of the equilibrium shape of the gas, the trapping frequency $\omega_{\perp}$ is thus replaced by the weaker value $\left(\omega_{\perp}^{2}-\Omega^{2}\right)^{1 / 2}$. One might have expected that a similar replacement should be done also for the monopole frequency; this is clearly not the case. For 


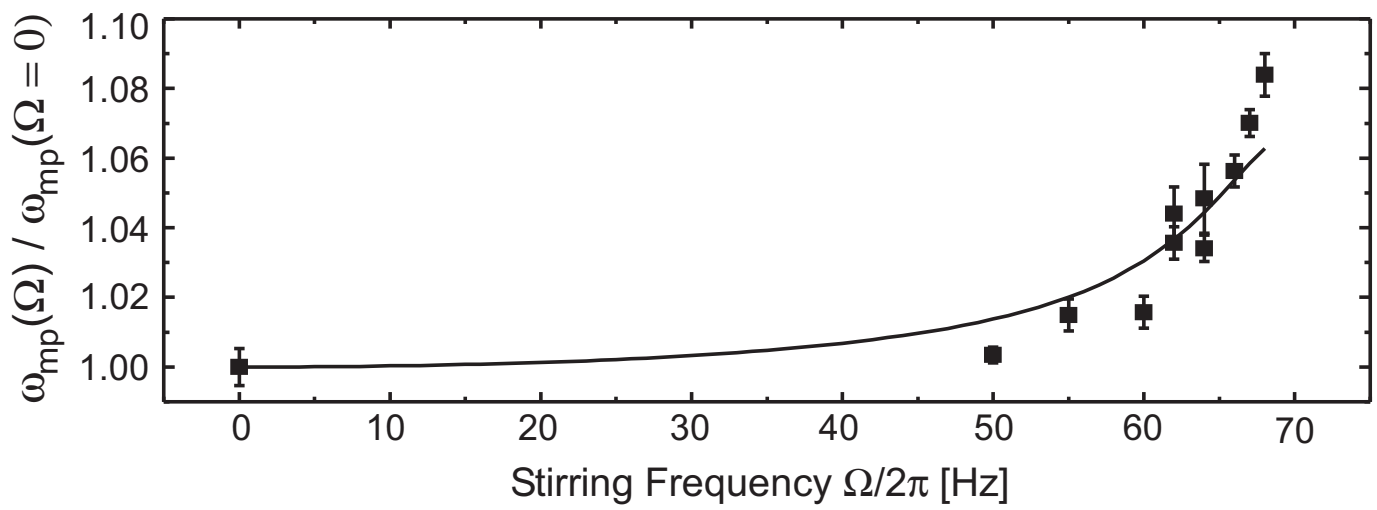

Fig. 2 - Transverse monopole frequency $\omega_{\mathrm{mp}}(\Omega)$ normalized by $\omega_{\mathrm{mp}}(\Omega=0)$ as a function of the stirring frequency $\Omega$. For a non rotating condensate $(\Omega=0)$ we measure $\omega_{\mathrm{mp}}(0) /(2 \pi)=130.6 \mathrm{~Hz}$. The vertical bars indicate the error given by the fitting routine.

a cigar-shaped condensate in the hydrodynamic regime, it was proven in [27] that $\omega_{0}$ stays equal to $2 \omega_{\perp}$ when $\Omega$ varies. In the case of an ideal gas described by classical mechanics, the equality $\omega_{\mathrm{mp}}=2 \omega_{\perp}$ also holds for any $\Omega$ as a consequence of the combined action of centrifugal and Coriolis forces.

(3) When the rotation frequency $\Omega$ approaches $\omega_{\perp}$, the contribution of the quartic term becomes more important and the monopole frequency $\omega_{\mathrm{mp}}$ deviates significantly from $2 \omega_{\perp}$. As shown in figure 2 this deviation reaches $\sim 8 \%$ for $\Omega /(2 \pi)=68 \mathrm{~Hz}$, i.e. $\Omega / \omega_{\perp} \simeq 1.05$. We have also plotted in figure 2 the prediction of a theoretical treatment of the monopole mode in the quartic + quadratic trap, neglecting the harmonic confinement along the $z$-direction. The agreement between these predictions and the experimental data is satisfactory, given the difference in geometry between the experiment and the model.

The starting point of our theoretical treatment is the set of equations for rotational hydrodynamics [27], which we write in the laboratory frame:

$$
\begin{aligned}
\partial_{t} \rho & =-\boldsymbol{\nabla} \cdot(\rho \boldsymbol{v}) \\
\partial_{t} \boldsymbol{v} & =-\boldsymbol{\nabla}\left((U+g \rho) / m+v^{2} / 2\right)+\boldsymbol{v} \times(\boldsymbol{\nabla} \times \boldsymbol{v}),
\end{aligned}
$$

where $\rho$ and $\boldsymbol{v}$ are the density and velocity field of the atom distribution. $U=m \omega_{\perp}^{2} r^{2} / 2+k r^{4} / 4$ stands for the trapping potential and $g=4 \pi \hbar^{2} a / m$ characterizes the strength of the atomic interactions ( $a$ is the scattering length). As explained in [29,27,30] these equations are valid when one is interested in a phenomenon whose characteristic length scale is larger than the distance between vortices.

The eigenmodes are obtained by linearizing (12) around the rotating equilibrium solution

$$
\boldsymbol{v}_{\mathrm{eq}}=\boldsymbol{\Omega} \times \boldsymbol{r} \quad g \rho_{\mathrm{eq}}=\mu-U-U_{\mathrm{cen}},
$$

where $\mu$ is the chemical potential and $U_{\text {cen }}=-m \Omega^{2} r^{2} / 2$ the centrifugal potential. We eliminate the velocity field to get a closed equation for the density variation $\delta \rho$, assuming an oscillation at frequency $\omega$ :

$$
-\nabla \cdot\left(\rho_{\mathrm{eq}} \nabla[\delta \rho]\right)=\frac{m}{g}\left(\omega^{2}-4 \Omega^{2}\right)[\delta \rho] .
$$


In absence of a quartic term the eigenfrequencies for the $m=0$ modes are

$$
\omega_{n}^{2}=4 \Omega^{2}+2 n(n+1)\left(\omega_{\perp}^{2}-\Omega^{2}\right), \quad n \text { positive integer, }
$$

and the corresponding eigenmodes are polynomials of degree $n$ with respect to $r^{2}$. For $n=1$, we recover in particular $\omega_{1} \equiv \omega_{\mathrm{mp}}=2 \omega_{\perp}$.

When $\Omega$ approaches $\omega_{\perp}$, all transverse modes $m=0$ become degenerate. Such a macroscopic degeneracy is reminiscent of the degeneracy of the energy levels of a single particle in a uniform magnetic field, leading to the well known Landau level structure 31. An equivalent degeneracy occurs, still at the single particle level, for an isotropic 2D harmonic oscillator of frequency $\omega_{\perp}$, considered in a frame rotating at frequency $\Omega=\omega_{\perp}$ [32. However we emphasize that our result here holds not for a single particle spectrum, but for the eigenmodes of a $N$-body system treated in the mean-field approximation. The occurrence of such a macroscopic degeneracy raises interesting questions concerning the linear response of the rotating system to an arbitrary excitation.

When the quartic potential is present, the above degeneracy is lifted. We set $x=r / R$ in (4) with $R=(4 \mu / k)^{1 / 4}$, to get the dimensionless eigenvalue equation for the $m=0$ modes:

$$
-\frac{1}{x} \frac{d}{d x}\left(x\left(1-\epsilon x^{2}-x^{4}\right) \frac{d[\delta \rho]}{d x}\right)=\Lambda(\epsilon)[\delta \rho] \quad \text { where } \quad \epsilon=\frac{m R^{2}}{2 \mu}\left(\omega_{\perp}^{2}-\Omega^{2}\right) .
$$

For each $\Omega$, i.e. for each $\epsilon$, we are interested in the lowest eigenvalue $\Lambda_{0}(\epsilon)$ of the hermitian operator in the left hand side of (6). We can then deduce the frequency of the lowest transverse monopole mode which is plotted as a continuous line in figure 2

$$
\omega_{\mathrm{mp}}^{2}=4 \Omega^{2}+\frac{\mu \Lambda_{0}(\epsilon)}{m R^{2}} .
$$

The quantity $\Lambda_{0}(\epsilon)$ is an increasing function of $\epsilon$ which we calculate numerically using a variational method with polynomial trial functions. Just at the critical rotation $\Omega=\omega_{\perp}$, $\epsilon=0$ and we get $\Lambda(0) \simeq 11.5$. The slow rotation limit corresponds to $\epsilon \gg 1$, in which case $\Lambda(\epsilon) \simeq 8 \epsilon ;$ we then recover the result $\omega_{\mathrm{mp}}=2 \omega_{\perp}$.

This analysis also explains the periodic apparition of a hole at the center of the density profile for $\Omega \sim \omega_{\perp}$, as observed in figure 1] The stationary density profile (3) varies as $r^{4}$ around the origin, whereas the mode $\delta \rho$ varies as $r^{2}$ in this region. The curvature of the density profile is therefore dominated by the phase of the perturbation.

For $\Omega \geq \omega_{\perp}$ and a relatively strong excitation $\left(I^{\prime} / I=4\right)$, we observe that the time evolution of the mode structure becomes asymmetric (see figure 3 this asymmetry is also slightly visible in the last row of figure 1). It consists of a periodic entering positive density wave, which starts on the edge of the condensate and gradually moves to the center in a time period $\sim \pi / \omega_{\perp}$. A possible interpretation of this unusual structure is that two (or more) transverse modes $m=0$ are simultaneously excited with a non-zero relative phase. Since these modes have similar frequencies (cf. Eq. (5) ) the initial phase difference between them stays nearly constant, and this can give rise to the observed phenomenon.

To summarize we have presented in this paper a detailed study of the transverse monopole mode of a fast rotating degenerate Bose gas. We have shown that the non-harmonic character of the potential (which is essential for the confinement of the gas) has a clear influence on the mode frequency. We have also given a simple analysis of this mode for an infinitely long condensate which is in good agreement with the experimental data.

Theoretical studies have shown that the addition of a quartic term in the harmonic potential may lead to the formation of a "giant vortex", i.e. a vortex with a circulation larger 


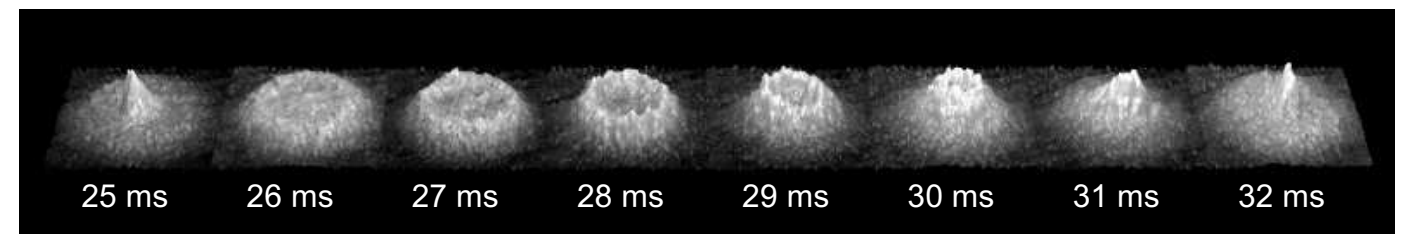

Fig. 3 - Time evolution of the density profile of the oscillating cloud, for $\Omega / 2 \pi=68 \mathrm{~Hz}$.

than the single quantum $h / m$ [18, 19,20,21, 22, 33. The large hole appearing at the center of the condensate during the monopole oscillation when $\Omega \gtrsim \omega_{\perp}$ should not be confused with such a giant vortex. We are dealing here with a transient state of the condensate, while the predicted giant vortex state is a stationary state of the system, for an appropriate angular momentum. Another example for a transient large core in a condensate is provided by an experiment recently performed in Boulder, in which a hole pierced in a rotating condensate confined in a purely quadratic trap was shown to persist for a long time 34.

We now briefly discuss some perspectives opened by this work. First we recall that the nature of the gas when the rotation frequency $\Omega$ is around or above $\omega_{\perp}$ is still unclear. The detailed study of 23] showed that the number of visible vortices is not sufficient to account for the measured rotation frequency of the gas. Two classes of explanation have been proposed to account for this finding. Either the fast rotating gas cannot be described anymore by a single macroscopic wave function [35, or the vortices are still present but they are distorted and do not show up clearly in the images of the condensate. We hope that the present experimental measurement of the transverse mode frequency can be used to discriminate between these two hypotheses. In principle a generalization of the above theoretical treatment to a condensate in a $3 \mathrm{D}$ trap is possible, and the quantitative predictions of such a model could be compared with the experimental findings.

From the experimental point of view a natural extension of the present work is to switch to a two-dimension geometry, using a strong confining potential along the rotation axis. Vortices then become point objects and the predicted properties of the system depend on the ratio between the atom number $N$ and the number of vortices $N_{v}$. When $N$ is large, the BoseEinstein condensate presents a regular vortex array, as already observed in [16]. The array is expected to melt when $N$ decreases to a value of the order of $N_{v}$ [36] (see also [11]). For $N<N_{v}$ the quantum Hall regime for particles should emerge. It is an interesting problem to determine the signature of these various regimes on the eigenmodes of the system. For the monopole mode considered here and for a pure harmonic confinement, it has been predicted in 37,38 that the frequency $\omega_{\mathrm{mp}}$ of the breathing motion of a $2 \mathrm{D}$ gas remains strictly equal to $2 \omega_{\perp}$ for any equilibrium state. This does not hold anymore in presence of the quartic term, and thus the deviation of $\omega_{\mathrm{mp}}$ from $2 \omega_{\perp}$ can in principle be used to monitor the emergence of new quantum phases in the rotating gas.

$$
* * *
$$

We thank S. Stringari and the ENS group for useful discussions. S. Stock acknowledges support from the European Union (CQG network HPRN-CT-2000-00125), the Studienstiftung des deutschen Volkes and the DAAD. This work is partially supported by CNRS, Collège de France, Région Ile de France, and DRED. 


\section{REFERENCES}

[1] R.J. Donnelly, Quantized Vortices in Helium II, Cambridge (1991), Chaps. 4 and 5.

[2] M. Tinkham, Introduction to superconductivity, McGraw-Hill (1996).

[3] M. R. Matthews, B. P. Anderson, P. C. Haljan, D. S. Hall, C. E. Wieman and E. A. Cornell, Phys. Rev. Lett., 83 (1999) 2498.

[4] K. W. Madison, F. Chevy, W. Wohlleben and J. Dalibard, Phys. Rev. Lett., 84 (2000) 806.

[5] J. R. Abo-Shaeer, C. Raman, J. M. Vogels and W. Ketterle, Science, 292 (2001) 476.

[6] E. Hodby, G. Hechenblaikner, S. A. Hopkins, O. M. Maragò and C. J. Foot, Phys. Rev. Lett., 88 (2002) 010405.

[7] N.R. Cooper, N.K. Wilkin and J.M.F. Gunn, Phys. Rev. Lett., 87 (2001) 120405.

[8] B. Paredes, P. Fedichev, J. I. Cirac, And P. Zoller, Phys. Rev. Lett., 87 (2001) 010402.

[9] T.-L. Ho, Phys. Rev. Lett., 87 (2001) 060403.

[10] N. Regnault and Th. Jolicceur, Phys. Rev. Lett., 91 (2003) 030402.

[11] U.R. Fischer, P.O. Fedichev and A. Recatti, cond-mat/0212419 Preprint (2002).

[12] N. Read And N.R. Cooper, cond-mat/0306378 Preprint (2003).

[13] G. BAym and C.J. Pethick, cond-mat/0308325 Preprint (2003).

[14] L.O. Baksmaty, S.J. Woo, S. Choi and N. Bigelow, cond-mat/0310567 Preprint (2003).

[15] I. Coddington, P. Engels, V. Schweikhard, E. A. Cornell, Phys. Rev. Lett., 91 (2003) 100402.

[16] V. Schweikhard, I. Coddington, P. Engels, V. P. Mogendorff and E. A. Cornell, cond-mat/0308582 Preprint (2003).

[17] A. L. Fetter, Phys. Rev. A, 64 (2001) 063608.

[18] E. Lundh, Phys. Rev. A, 65 (2002) 043604.

[19] K. Kasamatsu, M. Tsubota, and M. Ueda, Phys. Rev. A, 66 (2002) 053606.

[20] G.M. Kavoulakis and G. Baym, New Jour. Phys., 5 (2003) 51.1.

[21] M. Tsubota, K. Kasamatsu and T. Araki, cond-mat/0309364 Preprint (2003).

[22] A. Aftalion and I. Danila, cond-mat/0309668 Preprint (2003).

[23] V. Bretin, S. Stock, Y. Seurin and J. Dalibard, cond-mat/0307464 Preprint (2003).

[24] For a review, see e.g. F. Dalfovo, S. Giorgini, L.P. Pitaevski and S. Stringari, Rev. Mod. Phys., 71 (1999) 463.

[25] C. Fort, M. Prevedelli, F. Minard, F. S. Cataliotti, L. Ricci, G. M. Tino and M. Inguscio, Europhys. Lett., 49 (2000) 8.

[26] F. Chevy, V. Bretin, P. Rosenbusch, K. W. Madison, and J. Dalibard, Phys. Rev. Lett., 88 (2002) 250402.

[27] M. Cozzini and S. Stringari, Phys. Rev. A, 67 (2003) 041602.

[28] As for the dipole mode there is actually a small correction $(\sim 1 \%)$ to this relation due to the presence of the quartic potential $k r^{4} / 4$.

[29] A. Sedrakian and I. Wasserman, Phys. Rev. A, 63 (2001) 063605.

[30] F. Chevy and S. Stringari, cond-mat/0305559 Preprint (2003).

[31] L.D. Landau And E.M. Lifchitz, Quantum Mechanics, Course of Theoretical Physics, Vol.3, Chap. 15, Butterworth-Heinemann, Stoneham, MA (1981).

[32] In a frame rotating at angular velocity $\Omega$ the single particle Hamiltonian becomes $H^{\prime}=H-L_{z}$. Therefore all the levels of the $2 \mathrm{D}$ harmonic oscillator with an angular momentum $m$ equal to the ecitation number $n$ have an energy $n \hbar\left(\omega_{\perp}-\Omega\right)$ that goes to zero when $\Omega=\omega_{\perp}$.

[33] U.R. Fischer and G. Baym, Phys. Rev. Lett, 90 (2003) 140402.

[34] P. Engels, I. Coddington, P. C. Haljan, V. Schweikhard and E. A. Cornell, Phys. Rev. Lett, 90 (2003) 170405.

[35] E. Akkermans and S. Ghosh, cond-mat/0307418 Preprint (2003).

[36] J. Sinova, C.B. Hanna, and A.H. MacDonald, Phys. Rev. Lett., 89 (2002) 030403.

[37] L.P. Pitaevskil and A. Rosch, Phys. Rev. A, 55 (1997) R853.

[38] Yu. Kagan, E. L. Surkov and G. V. Shlyapnikov, Phys. Rev. A, 54 (1996) R1753. 